\title{
Optionen für eine Reform der Pflegeversicherung
}

\section{Heinz Rothgang und Maike Preuss}

Die jüngsten Vereinbarungen der großen Koalition in Berlin können die Finanzierungsprobleme der gesetzlichen Pflegeversicherung nicht dauerhaft lösen. Dafür sind grundsätzliche zwei Reformstrategien denkbar: ein Systemwechsel oder die Weiterentwicklung des bestehenden Systems der umlagefinanzierten sozialen Pflegeversicherung.

In den ersten Jahren ihres Bestehens hat die Pflegeversicherung Überschüsse erwirtschaftet. Seit 1999 jedoch ergeben sich steigende Defizite. Das geringere Defizit des Jahres 2005 resultiert aus den Zusatzeinnahmen durch den Zusatzbeitrag für Kinderlose, der seit 2005 regelmäßig anfällt.

Der für das Jahr 2006 gemeldete Überschuss in Höhe von zirka 450 Millionen Euro erweckt zunächst den Anschein, als wäre der Trend gebrochen. Der Überschuss resultiert jedoch lediglich aus einer Vorverlegung der Fälligkeit der Sozialversicherungsbeiträge im Januar (Gesetz zur Änderung des Vierten und Sechsten Buches Sozialgesetzbuch vom 3. August 2005). Dies führte zu einem Zufluss von insgesamt 13 Beiträgen in diesem Jahr. Hierbei handelt es sich um einen Einmaleffekt, der zu dem positiven Ergebnis führt. Wird von diesem Effekt abgesehen, zeigt sich auch für 2006 ein hohes Defizit - die Finanzlage der Pflegeversicherung bleibt prekär.

\section{Reformnotwendigkeit}

Auch in Zukunft werden die Ausgaben der Pflegeversicherung steigen. Der demografische Wandel führt dazu, dass auch in den nächsten Dekaden mit einer zunehmenden Zahl von pflegebedürftigen Menschen gerechnet werden muss. Entsprechende Modellrechnungen gehen dabei von einer Steigerung der Zahl der Pflegebedürftigen um 60 bis 80 Prozent aus (vgl. hierzu z. B. Hof 2001; Dietz 2002; Rothgang 2002; Nachhaltigkeitskommission 2003 sowie die Übersichtsdarstellung in Deutscher Bundestag 2002). Diese Fallzahlensteigerung entspräche einer jährlichen Ausgabensteigerung von 1,2 bis 1,5 Prozent, also einem Betrag von derzeit rund einer Viertel Milliarde Euro.

Auch eine Änderung des Pflegebedürftigkeitsbegriffes hätte potenziell merkliche Effekte auf die Ausgaben der Pflegeversicherung. Das derzeitige verrichtungsbezogene Verständnis von Pflegebedürftigkeit im SGB XI unterliegt seit seiner Einführung massiver Kritik. Das Gesundheits-

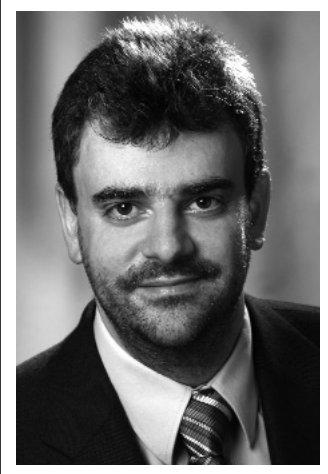

Prof. Dr. Heinz Rothgang ist Leiter der Abteilung Gesundheitsökonomie, Gesundheitspolitik und Versorgungsforschung des Zentrums für Sozialpolitik in Bremen. Außerdem ist er Inhaber des Lehrstuhls Gesundheitsökonomie des Fachbereiches Gesundheits- und Humanwissenschaften der Universität

Bremen.

E-Mail rothgang@zes.uni-bremen.de

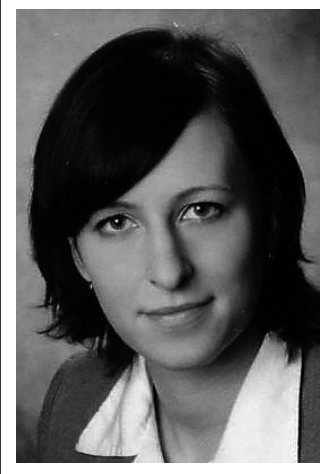

Maike Preuss ist Diplomökonomin und wissenschaftliche Mitarbeiterin mit dem Forschungsschwerpunkt Pflegeökonomie in der Abteilung Gesundheitsökonomie, Gesundheitspolitik und Versorgungsforschung des Zentrums für Sozialpolitik in Bremen. Sie lehrt an der Universität Bremen im Studiengang Public Health.

E-Mailmpreuss@zes.uni-bremen.de

ministerium hat daher einen Beirat eingesetzt, der Vorschläge für eine Neufassung des Pflegebedürftigkeitsbegriffs erarbeiten soll, der insbesondere eine verbesserte Erfassung des Betreuungsbedarfes Demenzkranker gewährleisten soll und damit zu einer Ausweitung der Zahl der Leistungsberechtigten und der Ausgaben der Pflegeversicherung führt.

Weiterhin belegen erste empirische Ergebnisse seit Einführung der Pflegeversicherung eine Veränderung des Inanspruchnahmeverhaltens Pflegebedürftiger, und zwar eine Nachfrageverschiebung vom ambulanten in den vollstationären Sektor, sowie einen Trend weg von rein informeller Pflege hin zu professioneller Pflege. Es wird erwartet, dass sich diese Trends in Zukunft fortsetzen (vgl. hierzu detailliert Blinkert/Klie 2001; Döhner/Rothgang 
2006). Dies wirkt bei den geltenden Leistungssätzen ausgabensteigernd.

Die Leistungshöhe der Hauptleistungsarten (Pflegegeld, Pflegesachleistungen, stationäre Pflege) ist seit Einführung der Pflegeversicherung unverändert geblieben. Bei steigenden Preisen für professionelle Pflege führt dies zu einem Kaufkraftverfall der Versicherungsleistungen und damit langfristig zur Delegitimierung der Pflegeversicherung. Eine Leistungsdynamisierung, die - soll die Kaufkraft der Versicherungsleistungen erhalten werden regelmäßig, regelgebunden und in Orientierung an der allgemeinen Lohnentwicklung erfolgen muss, ist daher notwendig.

Fallzahlsteigerungen, Verschiebungen der Pflegearrangements und eine angemessene Leistungsdynamisierung bewirken regelmäßig anfallende Leistungsausgabensteigerungen, die insgesamt leicht zu jährlichen Ausgabensteigerungen von bis zu vier Prozent führen können (vgl. Rothgang/Preuss 2007). Dazu kommt eine einmalige

Leistungsausweitung, sollte der Pflegebedürftigkeitsbegriff reformiert werden. Dem steht eine Steigerung der Bemessungsgrundlage gegenüber, die sich von 1997 bis 2004 auf durchschnittlich 0,8 Prozent belaufen hat. Selbst wenn für die Zukunft von einer größerer Steigerung der Bemessungsgrundlage ausgegangen wird, steht daher fest: Eine Finanzreform ist notwendig, soll eine weitere Absenkung der realen Pflegeversicherungsleistungen, die das System langfristig delegitimiert, und sollen zugleich dramatische Beitragssatzanstiege vermieden werden.

Diesen Notwendigkeiten folgt der Koalitionskompromiss vom 19. Juni 2007 zur Reform der Pflegeversicherung nur unzureichend (BMG 2007). Neben Leistungen zur Stärkung der ambulanten Versorgung nach persönlichem Bedarf sollen die Leistungen für Menschen mit erheblich eingeschränkter Alltagskompetenz auf bis zu 2.400 Euro jährlich angehoben werden. Diese Leistung soll auch für Menschen gewährt werden, denen keine Pflegestufe nach SGB XI zuerkannt wird. Ab dem Jahr 2015 sollen die Leistungen der Pflegeversicherung in einem dreijährigen Rhythmus in Anlehnung an die Inflation dynamisiert werden. Bis dahin ist eine stufenweise Erhöhung der ambulanten Sachleistungsbeträge, des Pflegegeldes, sowie der stationären Leistungsbeträge für die Pflegestufe III vorgesehen. Die Leistungen für Pflegebedürftige der Stufen I und II, die derzeit etwa 40 Prozent der Gesamtausgaben der Pflegeversicherung ausmachen, werden hingegen nicht erhöht. Insgesamt entsprechen die vorgesehenen Leistungsausweitungen damit nur einer durchschnittlichen Leistungsdynamisierung von jährlich 0,3 Prozent bezogen auf den Zeitraum 1996 bis 2012, bzw. 0,7 Prozent für den kommenden Zeitraum 2007 bis 2012. Diese Dynamisierung liegt also deutlich unter der Inflations- bzw. Lohnsteigerungsrate.

Die Finanzierung der geplanten Leistungserhöhungen soll durch eine Anhebung des Beitragssatzes um 0,25 Prozent zum 1. Juli 2008 erfolgen. Diese Erhöhung deckt gerade die geplanten Mehrausgaben bis zum Jahr 2014. Dabei bleiben die fiskalischen Mehrbedarfe, die sich aus der anstehenden Reform des Pflegebedürftigkeitsbegriffes ergeben, ebenso unberücksichtigt wie das aktuelle Defizit und die Tatsache, dass die Grundlohnsummensteigerung in den vergangenen Jahren nicht ausgereicht hat, um die demografisch bedingten und aus Veränderungen im Inanspruchnahmeverhalten resultierenden Mehrbedarfe abzudecken. Es ist daher zweifelhaft, ob die Finanzierung der Pflegeversicherung wirklich bis 2014 sichergestellt ist. Insgesamt erkaufen die aktuellen Pläne nur Zeit, langfristig ist eine umfassende Finanzierungsreform unumgänglich. Hierzu werden im Folgenden umfassendere Optionen diskutiert.

\section{Reformoptionen}

Für die Reform der gesetzlichen Pflegeversicherung lassen sich zwei grundsätzliche Reformstrategien unterscheiden: ein Systemwechsel oder die Weiterentwicklung des bestehenden Systems der umlagefinanzierten sozialen Pflegeversicherung.

- Ein Systemwechsel kann insbesondere erfolgen durch die Abschaffung der eigenständigen Pflegeversicherung und deren Integration in die gesetzliche Krankenversicherung, den Ersatz der Sozialversicherung durch ein steuerfinanziertes Leistungsgesetz oder durch den Umstieg auf eine kapitalfundierte (private)

Pflege(pflicht)versicherung. Die erstgenannte Variante wurde vom Sachverständigenrat zur Begutachtung der Entwicklung im Gesundheitswesen in seinem Jahresgutachten 2005 vorgeschlagen. Im Koalitionsvertrag legt sich die Regierung allerdings auf den Erhalt einer eigenständigen Pflegeversicherung fest. Die zweite Variante wurde in der Rürup-Kommission diskutiert und verworfen. Ihre Umsetzung ist somit ebenfalls unwahrscheinlich. Bereits seit Jahren in der Diskussion und nicht ohne weiteres als unrealistisch abzutun ist dagegen der Umstieg auf eine kapitalfundierte Versicherung, der insbesondere von Ökonomen oft mit der Begründung gefordert wird, dass dadurch ein höheres Wirtschaftswachstum oder eine höhere individuelle Rendite erzielt werden könne, dass kapitalfundierte Systeme weniger anfällig für den demografischen Wandel seien und dass sie die intergenerative Gerechtigkeit erhöhen würden (vgl. Rothgang 2004: 597 ff. für eine ausführlichere Darstellung). Abgesehen davon, dass erhebliche Zweifel daran bestehen, ob diese Vorteile realisiert werden können, wird der Umstieg auf ein kapitalfundiertes System ein Hauptproblem auf: Jede Art von Umstieg führt zu einer Zusatzbelastung der heutigen Beitragszahlergeneration, die nicht nur die Bedarfe der aktuellen Leistungsempfängergeneration finanzieren, sondern zusätzlich den Kapitalstock für ihre eigenen Ansprüche ansparen muss (Rothgang 2005b: 118). Die daraus resultierende intertemporale Lastenverschiebung von der Zukunft in die Gegenwart kann angesichts der ökonomischen Lage als nicht wünschenswert angesehen werden (BMGS 2003: S. 215 f.) besonders unter Berücksichtigung der jüngsten Entwicklungen in der Altersvorsorge. Bereits hier wird von den heute Erwerbstätigen eine verstärkte private Vorsorge erwartet, die das verfügbare Einkommen belastet. Selbst bei der Wahl eines »Auslaufmodells«, bei dem auf einen sofortigen Umstieg verzichtet und nur der jüngere Teil der Bevölkerung in ein kapitalfundiertes System einbezogen wird, kommt es für weite Teile der Bevölkerung zu erheblichen Härten, sodass Umstiegs- 
modellen letztlich allein schon aus diesem Grund eine Absage erteilt werden muss.

- Soll das bisherige umlagefinanzierte System der Pflegeversicherung erhalten bleiben, müssen zusätzliche Finanzmittel erschlossen werden. Dies ließe sich am einfachsten durch eine Anhebung des Beitragssatzes realisieren, wie sie jetzt auch von der großen Koalition vereinbart wurde. Eine solche Anhebung war bereits in der Begründung des Entwurfs des Pflege-Versicherungsgesetzes vorgesehen. Beläuft sich diese Anhebung auf wenige Zehntel Beitragssatzpunkte ist sie sicherlich makroökonomisch unproblematisch. Etwaigen Bedenken wegen erhöhter Lohnzusatzkosten könnten zudem durch ein Einfrieren des Arbeitgeberanteils Rechnung getragen werden. Allerdings ist eine Erhöhung um wenige Zehntel Beitragssatzpunkte langfristig nicht ausreichend. Würden die steigenden Ausgaben allein durch Beitragssatzsteigerungen finanziert, wäre in den nächsten drei Dekaden ansonsten nämlich eine Verdopplung des Beitragssatzes zu erwarten. Eine moderate Beitragssatzsteigerung kann daher nur ein Teilelement einer Mischstrategie sein.

Wie in der Renten- und Krankenversicherung könnte auch in der Pflegeversicherung ein steuerfinanzierter Bundeszuschuss oder steuerfinanzierte Beiträge eingeführt werden. Steuerfinanzierte Zuschüsse bedürfen in einem Sozialversicherungssystem einer Begründung. Eine solche Begründung wird in der Rentenversicherung darin gesehen, dass das Versicherungssystem allgemeine Staatsaufgaben als »versicherungsfremde Leistungen« übernimmt. Entsprechend sieht das GKV-Wettbewerbsstärkungsgesetz einen Anstieg der Steuerfinanzierung vor, um so die beitragsfreie Mitversicherung der Kinder in der gesetzlichen Krankenversicherung auszugleichen, die als allgemeine Staatsaufgabe angesehen wird, die - systemgerecht - aus Steuermitteln finanziert werden sollte. Mit derselben Argumentation könnten auch in der Pflegeversicherung Steuermittel eingesetzt werden. Allerdings liegt der Anteil der Leistungsempfänger im Alter von bis zu 20 Jahren, der als Anhaltspunkt für den Anteil der Ausgaben der beitragsfrei mitversicherte Kinder angesehen werden kann, bei unter fünf Prozent. Ein Bundeszuschuss zum Ausgleich der Kindermitversicherung hätte damit einen Umfang von deutlich unter einer Milliarde Euro und kann deshalb lediglich als ein Element einer umfassenden Strategie angesehen werden.

Die Einführung von Bürgerversicherungselementen stellt $\mathrm{ab}$ auf eine Ausweitung des versicherungspflichtigen Personenkreises und/oder die Einbeziehung weiterer Einkommen und Einkommensarten. Werden zusätzliche Einkommensarten, wie Kapitaleinkommen, einbezogen, erhöht dies nicht nur die Einnahmen der sozialen Pflegeversicherung, sondern baut gleichzeitig bestehende Ungleichheiten ab. Bisher zahlen Versicherte mit gleichem Einkommen, deren Einkommen sich aber zu verschiedenen Teilen aus Arbeits- oder Lohnersatzeinkommen und anderen Einkommensarten zusammensetzen, unterschiedliche Beiträge.

Die Anhebung der Beitragsbemessungsgrenze würde ebenfalls zu steigenden Einnahmen führen und die vertikale Umverteilung, von den Einkommensstarken zu den Einkommensschwachen, stärken. Würden darüber hinaus noch die bisher privat Versicherten in die soziale Pflegeversicherung einbezogen, würde dies zwar gleichzeitig zu einer Ausweitung des leistungsberechtigten Personenkreises führen, und damit Mehrkosten generieren, hätte aber nach Lauterbach et al. (2005: 24) dennoch per Saldo positive Auswirkungen auf die Finanzsituation der Pflegeversicherung. Bei den bislang Privatversicherten handelt es sich nämlich um so genannte "gute Risiken«, Versicherte mit überdurchschnittlichen Einkommen und/oder unterdurchschnittlichen Kosten. So lagen die durchschnittlichen Leistungsausgaben für einen gesetzlich Versicherten 2003 bei 248 Euro, die für einen privat Versicherten hingegen bei 58 Euro. (Diese von Lauterbach et al. [2005] genannten Werte berücksichtigen allerdings nicht, dass rund die Hälfte der Privatversicherten beihilfeberechtigt ist und die resultierenden Beihilfeausgabe für einen Systemvergleich hinzuaddiert werden müssten. Auch nach einer solchen Korrektur sind die Ausgaben für die gesetzliche Versicherten aber etwa drei mal so hoch wie für die privat Versicherten.)

Die Einführung von Bürgerversicherungselementen unterstützt das Prinzip einer solidarischen Pflegeversicherung, in welcher sich möglichst alle Menschen beteiligen, und dabei je nach persönlicher Leistungsfähigkeit belastet werden. Auch ihre fiskalische Ergiebigkeit ist allerdings begrenzt. So berechnen Lauterbach et al. unter Rückgriff auf durchaus optimistische Annahmen Mehreinnahmen im Umfang von 0,2 bis 0,5 Beitragssatzpunkten - bei einem Mehrbedarf, der bis 2040 bei rund zwei Beitragssatzpunkten liegt. Auch die Bürgerversicherung kann daher nur ein Element einer umfassenden Strategie sein.

\section{Reformoptionen im Überblick}

Grundsätzlich stehen für die Reform der gesetzlichen Pflegeversicherung zwei Reformstrategien zur Verfügung: ein Systemwechsel oder die Weiterentwicklung des bestehenden Systems der umlagefinanzierten sozialen Pflegeversicherung - mit unterschiedlichen Folgen:

\section{Systemwechsel:}

- Abschaffung der Pflegeversicherung zugunsten eines steuerfinanzierten Leistungsgesetzes

- Umstieg auf ein kapitalfundiertes System

Erhalt und Ausbau des bestehenden Systems: Leistungsdynamisierung und Erschließung neuer Finanzierungsquellen durch

- Beitragssatzerhöhung

- steuerfinanzierte Bundeszuschüsse oder steuerfinanzierte Beiträge

- Bürgerversicherung(selemente)

- ergänzende Kapitaldeckung durch

- Kopfprämien für alle

- Zusatzbeitrag für Rentner plus Vorsorgepflicht für Beschäftigte

Heinz Rothgang/Maike Preuss 
Schließlich kann eine ergänzende Kapitalbildung betrieben werden. Diese kann beispielsweise erfolgen, indem alle Versicherten verpflichtet werden, in ein ergänzendes System einzuzahlen. Dies ist vom PKV-Verband 2005 und von der bayerischen Sozialministerin vorgeschlagen worden, wobei jährlich steigende Kopfprämien vorgesehen sind, die zur Finanzierung der Leistungsdynamisierung und dem Aufbau eines Kapitalstocks verwendet werden sollen. Die Ausgestaltung als Kopfprämienmodell führt im Vergleich zu einem System einkommensabhängiger Beiträge - zu einer Umverteilung von unten nach oben, die anders als in den Kopfprämienmodellen zur Krankenversicherung nicht durch Zuschüsse für einkommensschwache Haushalte abgefedert werden soll. Zudem wird mit der Zusatzversicherung ein weiteres Leistungssystem aufgebaut. Das zieht zusätzliche Verwaltungskosten und Koordinierungsprobleme nach sich, insbesondere wenn das Zusatzsystem im Zeitverlauf quantitativ immer mehr an Bedeutung gewinnt.

Wenn ein Kapitalaufbau gewünscht wird, um so zukünftige Lasten in die Gegenwart vorzuziehen, so erscheint eine Kapitalbildung im bestehenden System überlegen. Allerdings kann eine derartige »Demografiereserve«, wenn sie einmal aufgebaut ist, leicht für andere Zwecke missbraucht werden. Dem könnte entgegengetreten werden, wenn der Kapitalaufbau mit einem Zusatzbeitrag für Rentner kombiniert wird. Ein solcher in der Rürup-Kommission diskutierter Zusatzbeitrag lässt sich rechtfertigen, da die jetzigen Rentner in den Genuss der Leistungen der Pflegeversicherung kommen, ohne jahrzehntelange Vorleistungen erbracht zu haben, wie es bei den nachwachsenden Kohorten der Fall ist. Der Zusatzbeitrag könnte so die intergenerative Gerechtigkeit erhöhen. Die nachwachsenden Kohorten müssten verpflichtet werden, für diesen Zusatzbeitrag durch individuelle Kapitalbildung vorzusorgen. Damit wäre ein Element der Kapitalbildung eingeführt, zugleich würden durch den Zusatzbeitrag aber unmittelbar Einnahmen an die Pflegeversicherung fließen. Allerdings kann ein solches Element nicht befürwortet werden, ohne zu prüfen, wie stark die Rentner zugleich durch andere sozialpolitische Maßnahmen, insbesondere im Bereich der Alterssicherung, jüngst belastet wurden und noch belastet werden.

\section{Fazit}

Das bisherige System der Pflegeversicherung ist reformfähig. Soll eine Delegitimierung vermieden werden, müssen seine Leistungen regelgebunden dynamisiert werden. Dies setzt voraus, dass weitere Finanzierungsquellen erschlossen werden. Hierbei ist eine Kombination verschiedener Maßnahmen zur Einnahmensteigerung möglich und sinnvoll. Werden Bürgerversicherungselemente mit einer moderaten Beitragssatzsteigerung und steuerfinanzierten Elementen verbunden und eventuell durch eine ergänzende Kapitalfundierung komplettiert, könnte diese Kombination die zukünftige Finanzierung der Pflegeversicherung sicherstellen. Diese Strategie ist einer Abkehr vom umlagefinanzierten System und einer damit verbundenen Einführung eines kapitalgedeckten Systems vorzuziehen, da der Systemwechsel sozial unverträgliche Umstellungskosten generiert und darüber hinaus gehende erwünschte Effekte zweifelhaft sind.

\section{Literatur}

Blinkert, Baldo; Klie, Thomas (2001): Zukünftige Entwicklung des Verhältnisses von professioneller und häuslicher Pflege bei differierenden Arrangements und privaten Ressourcen bis zum Jahr 2050, Expertise im Auftrag der Enquete-Kommission Demografischer Wandel des Deutschen Bundestages, Freiburg im Breisgau/Wiesbaden, März 2001. Internet http://www.soziologie.uni-freiburg.de/fifas/all/pdf/Expertise Bundestag.pdf, 03.11.2006.

BMGS (=Bundesministerium für Gesundheit und Soziale Sicherung) (Hg.) (2003): Nachhaltigkeit in der Finanzierung der sozialen Sicherungssysteme. Bericht der Kommission. Berlin: Eigenverlag.

BMG (= Bundesministerium für Gesundheit) (2006): BMG Statistiken Pflege, in Zahlen: Daten zur Pflegeversicherung: Internet http://www.bmg.bund.de/cln_040/nn_604244/DE/Themenschwerpunkte/Pflegeversicherung/Zahlen-und -Fakten/ zahlen-und-fakten-node, param $=$.html_nnn=true.

BMG (= Bundesministerium für Gesundheit) (2007): Reform zur nachhaltigen Weiterentwicklung der Pflegeversicherung, 19. Juni 2007. Internet

http://www.bmg.bund.de/cln_040/nn_600110/DE/Themenschwerpunkte/Pflegeversicherung/pflegerefo rmdownload,templateId=raw, property=publicationFile.pdf/ pflegereform-download.pdf, 30.6.2007.

Deutscher Bundestag (2002): Schlussbericht der Enquete-Kommission $»$ Demografischer Wandel - Herausforderungen unserer älter werdenden Gesellschaft an den einzelnen und die Politik." Bundestags-Drucksache 14/8800 vom 28. März 2002.

Dietz, Berthold (2002): Die Pflegeversicherung: Ansprüche, Wirklichkeiten und Zukunft einer Sozialreform, Westdeutscher Verlag: Wiesbaden.

Döhner, Hanneli; Rothgang, Heinz (2006): Pflegebedürftigkeit. Zur Bedeutung der familialen Pflege für die Sicherung der Langzeitpflege, in: Bundesgesundheitsblatt - Gesundheitsforschung - Gesundheitsschutz, Band 6, p. 583-594.

Hof, Bernd (2001): Auswirkungen und Konsequenzen der demografischen Entwicklung für die gesetzliche Kranken- und Pflegeversicherung. Gutachten im Auftrag des Gesamtverbandes der Deutschen Versicherungswirtschaft e. V. und des Verbandes der privaten Krankenversicherung e. V., Institut der deutschen Wirtschaft: Köln, Internet http://www.pkv.de/ downloads/Doku24.pdf, 09.03.2007.

Lauterbach, Karl; Lüngen, Markus; Stollenwerk, Björn; Gerber, Andreas; Klever-Deichert, Gabriele (2005): Auswirkungen einer Bürgerversicherung in der Pflegeversicherung, unveröff. Ms. Eine Kurzfassung wurde veröffentlich in: Gesundheitsökonomie und Qualitätsmanagement: 221-230.

Nachhaltigkeitskommission (= Kommission für die Nachhaltigkeit in der Finanzierung der sozialen Sicherungssysteme) (2003): Bericht der Kommission. Berlin: Bundesministerium für Gesundheit und soziale Sicherung.

Rothgang, Heinz (2002): Finanzwirtschaftliche und strukturelle Entwicklungen in der Pflegeversicherung bis 2040 und mögliche alternative Konzepte. Endbericht zu einer Expertise für die Enquete-Kommission »Demografischer Wandel« des Deutschen Bundestages, in: Enquete-Kommission »Demografischer Wandel« (Hg.): Herausforderungen unserer älter werdenden Gesellschaft an den einzelnen und die Politik - Studienprogramm, Band V, R. v. Decker Verlag: Heidelberg.

Rothgang, Heinz (2004): Reformoptionen zur Finanzierung der Pflegesicherung. Darstellung und Bewertung. In: Zeitschrift für Sozialreform, 50. Jg., Heft 6, S. 584-616.

Rothgang, Heinz (2005a): Demografischer Wandel und Pflege(ver)sicherung, in: Kerschbaumer, Judith / Schröder, Wolfgang (Hg.): Sozialstaat und demografischer Wandel. Herausforderungen für Arbeitsmarkt und Sozialversicherung, Wiesbaden: VS Verlag für Sozialwissenschaften, 119-146.

Rothgang, Heinz (2005b): Finanzbedarf und Finanzierungsoptionen für eine Reform der Pflegeversicherung, in: Soziale Sicherheit, Heft 4: 114-121.

Rothgang, Heinz/Preuss, Maike (2007): Was können wir über Finanzierungsprobleme der sozialen Pflegeversicherung in der Zukunft wissen? in: Igl, Gerhard (Hg.): Reform der Pflegeversicherung, im Erscheinen.

Statistisches Bundesamt (2003): Bevölkerung Deutschlands bis 2050. 10. koordinierte Bevölkerungsvorausberechnung, Statistisches Bundesamt: Wiesbaden. 